\title{
Semiclassical force for electroweak baryogenesis: Three-dimensional derivation
}

\author{
Kimmo Kainulainen, ${ }^{1, *}$ Tomislav Prokopec, ${ }^{2, \dagger}$ Michael G. Schmidt, ${ }^{2, \sharp}$ and Steffen Weinstock ${ }^{2, \S}$ \\ ${ }^{1}$ Theory Division, CERN, CH-1211, Geneva, Switzerland \\ and NORDITA, Blegdamsvej 17, DK-2100, Copenhagen $Ф$, Denmark \\ ${ }^{2}$ Institute for Theoretical Physics, Heidelberg University, Philosophenweg 16, D-69120 Heidelberg, Germany
}

(Received 19 February 2002; published 5 August 2002)

\begin{abstract}
We derive a semiclassical transport equation for fermions propagating in the presence of a $C P$-violating planar bubble wall at a first order electroweak phase transition. Starting from the Kadanoff-Baym (KB) equation for the two-point (Wightman) function we perform an expansion in gradients, or equivalently in the Planck constant $\hbar$. We show that to first order in $\hbar$ the KB equations have a spectral solution, which allows for an on-shell description of the plasma excitations. The $C P$-violating force acting on these excitations is found to be enhanced by a boost factor in comparison with the $(1+1)$-dimensional case studied in a former paper. We find that an identical semiclassical force can be obtained by the WKB method. Applications to the MSSM are also mentioned.
\end{abstract}

DOI: 10.1103/PhysRevD.66.043502

PACS number(s): $98.80 . \mathrm{Cq}$

\section{INTRODUCTION}

Modeling electroweak baryogenesis (EWBG) [1] requires a study of the generation and transport of $C P$-violating flows arising from interactions of fermions with the expanding phase transition fronts. Indeed, the most prominent problem in the EWBG scenario over the past few years has been to find a systematic derivation of the appropriate transport equations [2], including the $C P$-violating sources. This paper is the second in a series dedicated to reach this goal. In the first paper [3] [Kainulainen-Prokopec-Schmidt-Weinstock paper I (KPSW-I)] we derived a semiclassical transport equation starting with the Kadanoff-Baym (KB) equations [4,5] for the Wightman out-of-equilibrium two-point function. For simplicity we have restricted ourselves to $1+1$ dimensions there. An important tool for the derivation was an expansion in gradients in the center-of-mass coordinate. This is a good approximation when the typical de Broglie wavelength $l_{\mathrm{dB}}$ $\sim 1 / T$ of a particle in the plasma is small in comparison to the wall thickness, $l_{\mathrm{dB}} \ll l_{\text {wall }}$. This is the case for example in many supersymmetric models, which typically have $l_{\text {wall }}$ $\sim(10-20) / T[6]$. Here we extend the derivation to the more realistic case of fermions interacting with a planar phase transition front in 3+1 dimensions. As in [3] we only consider collisionless fermions and we do not account for the gauge degree of freedom. Some aspects of collision terms were discussed in [7] and the gauge fields were studied in the $(1+1)$-dimensional case in [8].

A crucial step in our derivation is to show that, to second order in gradients or equivalently to first order in $\hbar$, the $\mathrm{KB}$ equation for the Wightman function $G^{<}$written in the mixed (Wigner) coordinates admits a spectral solution. This is not a

\footnotetext{
*On leave of absence from University of Jyväskylä, Finland. Email address: kimmo.kainulainen@cern.ch

†Email address: T.Prokopec@ uni-heidelberg.de

*Email address: M.G.Schmidt@uni-heidelberg.de

${ }^{\S}$ Email address: S.Weinstock@thphys.uni-heidelberg.de
}

generic feature, and does not persist beyond the order $\hbar$. Nevertheless, to first order in $\hbar$ one can write an effective kinetic equation for a suitably chosen distribution function for the corresponding on-shell excitations. This kinetic equation has the familiar Liouville form, but with a semiclassical force term that includes a $C P$-violating part of order $\hbar$ which sources baryogenesis. This $C P$-violating force is in agreement with the earlier results [9] derived by use of WKBmethods [10-12].

It should be stressed that the existence of the spectral solution to order $\hbar$ is a new result. Indeed, while fermion dynamics in the presence of classical background fields have been studied extensively within the Schwinger-Keldysh formalism [13-17], apparently no attempts have been made to find explicit solutions beyond the classical approximation, nor does the fact that the spectral decomposition ansatz fails beyond the order $\hbar$ seem to be known. Our result is also crucial for EWBG-calculations since no $C P$-violating terms appear in the classical mean field approximation. Moreover, as the failure of the spectral decomposition ansatz beyond order $\hbar$ means that higher order effects cannot be described in the semiclassical limit, we are very lucky that in the most interesting cases for the EWBG the walls are thick enough so that the gradient expansion converges rapidly and our semiclassical theory can be expected to be a very good approximation.

This paper is organized as follows. In Sec. II we derive the Kadanoff-Baym equation for a collisionless fermionic system and introduce the Lorentz-boost that relates the wall rest frame and the local frame with no parallel momentum, where the $\mathrm{KB}$ equations can be reduced to the $(1+1)$-dimensional form. We then construct an explicit connection between the two frames and make use of our old $(1+1)$-dimensional results [3] to derive the semiclassical transport equations appropriate for the EWBG-problem in $3+1$ dimensions. In Sec. III we consider the transformation properties of fermionic currents and relate the $C P$-violation in the fluid equations to the non-conservation of the axial current divergence. In Sec. IV we derive the semiclassical force in the WKB-approach and in Sec. V we apply our 
results to chargino transport in the MSSM. Finally, Sec. IV contains our conclusions.

\section{DERIVATION OF KINETIC EQUATIONS}

As in KPSW-I [3], we will consider a fermionic field described by the Lagrangian

$$
\mathcal{L}=i \bar{\psi} b \psi-\bar{\psi}_{L} m \psi_{R}-\bar{\psi}_{R} m^{*} \psi_{L}+\mathcal{L}_{\text {int }},
$$

where the mass

$$
m(u)=m_{R}(u)+i m_{I}(u)=|m(u)| \mathrm{e}^{i \theta(u)}
$$

arises from an interaction with a spatially varying scalar field condensate. This situation is realized at the first order electroweak phase transition (EWPT), which proceeds via nucleation and growth of the broken phase bubbles of a nonzero Higgs condensate. EWPT bubbles quickly grow very large in comparison with the thickness of the phase transition front, so one can to a good approximation assume a planar symmetry on the scale of the bubble walls. As a result we can take $m$ to be a function of one spatial coordinate. Moreover, bubble walls are often found to be very wide [6] in comparison with the typical de Broglie wavelength of a particle in the plasma, $l_{\text {wall }} \ll l_{\mathrm{dB}} \sim 1 / T$. This suggests that one should be able to treat the kinetics of plasma excitations near the phase transition front in an expansion in gradients.

Here we consider only a collisionless plasma. Interactions are specified by the Lagrangian $\mathcal{L}_{\text {int }}$, and in addition to reproducing the usual collision terms in kinetic theory, they give rise to a new $C P$-violating source [8]. We will study these effects in detail elsewhere [18]. For simplicity we also restrict ourselves to a case without gauge degrees of freedom. Transport equations with a self-consistent electric field were considered in $1+1$ dimensions in Ref. [8].

As in [3], the basic object of our study is the dynamical two point (Wightman) function

$$
G_{\alpha \beta}^{<}(u, v)=i\left\langle\bar{\psi}_{\beta}(v) \psi_{\alpha}(u)\right\rangle,
$$

where the expectation value is taken over the initial state. In the collisionless case the equation of motion for $G^{<}$can be obtained by use of the Dirac equation that follows from the Lagrangian (1):

$$
\left[i b_{u}-m_{R}(u)+i m_{I}(u) \gamma_{5}\right] \psi(u)=0 .
$$

In order to establish the gradient expansion, we first separate the average center-of-mass coordinate $x=(u+v) / 2$ from the relative coordinate $r=u-v$ by performing the Wigner transform (Fourier transform with respect to $r$ ) to the mixed representation:

$$
G^{<}(k ; x)=\int d^{4} r e^{i k \cdot r} G^{<}(x+r / 2, x-r / 2) .
$$

For further reference we note that $G^{<}(x, k)$ satisfies the Hermiticity property

$$
\left[i \gamma^{0} G^{<}\right]^{\dagger}=i \gamma^{0} G^{<}
$$

implied by the definition (3). Using Eq. (4) and transforming to the mixed representation, one finds the equation of motion

$$
\left[k+\frac{i}{2} \not \partial-m_{R}\left(x-\frac{i}{2} \partial_{k}\right)-i \gamma^{5} m_{I}\left(x-\frac{i}{2} \partial_{k}\right)\right] i G^{<}=0
$$

where

$$
m_{R, I}\left(x-\frac{i}{2} \partial_{k}\right) \equiv m_{R, I}(x) \mathrm{e}^{-(i / 2) \overleftarrow{t} \cdot \partial_{k}}
$$

Formally Eq. (7) is completely general and valid up to any order in gradients and for arbitrary space and time dependence of the mass term (8). This expansion in gradients of the center-of-mass coordinate $x$ can be also viewed as an expansion in powers of the Planck constant. We have set $\hbar$ $\rightarrow 1$, but a dimensionful $\hbar$ can at any stage be easily restored by the simple replacements $\partial_{x} \rightarrow \hbar \partial_{x}$ and $G^{<} \rightarrow \hbar^{-1} G^{<}$.

In the EWBG problem the system is both stationary and has planar symmetry along spacelike hypersurfaces of constant $z$ in the wall rest frame. This means that in the plasma rest frame the mass has the form

$$
m_{R, I}=m_{R, I}\left(\gamma_{w}\left(z-v_{w} t\right)\right)
$$

where $v_{w}$ is the velocity of the phase transition front and $\gamma_{w}$ the corresponding boost factor. It is easiest to perform the analysis in the wall rest frame where $m_{R, I}=m_{R, I}(z)$. Moreover, because Eq. (7) is Lorentz-covariant, one can immediately write the equations of motion in the wall frame. We also assume a stationary solution for $G^{<}$, so that its functional dependence can be parametrized in the wall frame as $G^{<}\left(k_{\mu} ; z\right)$ [19]. Keeping the gradient corrections still to arbitrary order the equation of motion (7) then becomes

$$
\begin{gathered}
{\left[\gamma^{0} k_{0}-\gamma^{3}\left(k_{z}-\frac{i}{2} \partial_{z}\right)-\vec{\gamma} \cdot \vec{k}_{\|}-m_{R}\left(z-\frac{i}{2} \partial_{k_{z}}\right)\right.} \\
\left.-i \gamma^{5} m_{I}\left(z-\frac{i}{2} \partial_{k_{z}}\right)\right] i G^{<}=0 .
\end{gathered}
$$

Despite these simplifications, Eq. (10) still appears quite formidable to solve. Indeed, the Wightman function $G^{<}$contains 16 independent real functions and Eq. (10) consists of 16 complex equations for these components [20]. However, when $\vec{k}_{\|} \rightarrow 0$, i.e. for particles that move orthogonally to the wall, Eq. (10) reduces to the much simpler $(1+1)$-dimensional one studied in [3]. We can make use of those results in the general case studied here if we can find a transform that removes the $\vec{\gamma} \cdot \vec{k}_{\|}$term from Eq. (10). In the static case under consideration this is done by the boost $\Lambda$ to the frame in which $\vec{k}_{\|}=0$. Obviously the four momentum in the boosted frame becomes 


$$
\overrightarrow{\tilde{k}}_{\|}=0, \quad \widetilde{k}_{z}=k_{z}, \quad \widetilde{k}_{0}=\operatorname{sgn}\left[\mathrm{k}_{0}\right]\left(k_{0}^{2}-\vec{k}_{\|}^{2}\right)^{1 / 2} .
$$

We define the boost velocity $\vec{v}_{\|}=\vec{k}_{\|} / k_{0}$ and the gammafactor $\gamma_{\|}=k_{0} / \widetilde{k}_{0}$ for later reference. Finding the spinor representation of the boost $\Lambda$ requires a little more work, but it can be deduced from the fact that the desired operator $L(\Lambda)$ must commute with $\gamma^{3}$ and $\gamma^{5}$ and it must effect the transform

$$
L(\Lambda) k L^{-1}(\Lambda)=\gamma^{0} \widetilde{k}_{0}-\gamma^{3} k_{z} \equiv \tilde{k} .
$$

The commutation requirements immediately suggest the form $L(\Lambda)=a+b \gamma^{0} \vec{\gamma} \cdot \vec{v}_{\|}$, and imposing the condition (12) then defines the coefficients $a$ and $b$, leading to the operator

$$
L(\Lambda)=\frac{k_{0}+\widetilde{k}_{0}-\gamma^{0} \vec{\gamma} \cdot \vec{k}_{\|}}{\sqrt{2 \widetilde{k}_{0}\left(k_{0}+\widetilde{k}_{0}\right)}} .
$$

The inverse boost operator is simply $L^{-1}\left(\Lambda\left(k_{0}, \vec{k}\right)\right)$ $=L\left(\Lambda\left(k_{0},-\vec{k}\right)\right)$. After performing the boost, we finally arrive at the following effectively $(1+1)$-dimensional equation:

$$
\begin{gathered}
{\left[\gamma^{0} \widetilde{k}_{0}-\gamma^{3}\left(k_{z}-\frac{i}{2} \partial_{z}\right)-m_{R}\left(z-\frac{i}{2} \partial_{k_{z}}\right)\right.} \\
\left.-i \gamma^{5} m_{I}\left(z-\frac{i}{2} \partial_{k_{z}}\right)\right] i \widetilde{G}^{<}=0,
\end{gathered}
$$

where the boosted Wigner function $\widetilde{G}^{<}$is related to the original one by

$$
\widetilde{G}^{<}=L(\Lambda) G^{<} L(\Lambda)^{-1} .
$$

The further analysis of Eq. (14) is identical to our earlier treatment [3]. However, for completeness we review the main physical aspects of the derivation here. The most important property of the $(1+1)$-dimensional frame is that the differential operator in Eq. (14) is entirely spanned by the $(1+1)$-dimensional Clifford algebra, and that it commutes with the operator

$$
\widetilde{S}_{z}=\gamma^{0} \gamma^{3} \gamma^{5}
$$

which measures spin $s$ in the $z$-direction in which the wall moves. Therefore $s$ is a good quantum number in the boosted frame, and as a result we can restrict ourselves to finding solutions for $\widetilde{G}^{<}$that satisfy $\widetilde{S}_{z} \widetilde{G}_{s}^{<}=\widetilde{G}_{s}^{<} \widetilde{S}_{z}=s \widetilde{G}_{s}^{<}$. This condition immediately leads to the following decomposition for $\widetilde{G}^{<}$, which is block-diagonal:

$$
-i \gamma^{0} \widetilde{G}_{s}^{<}=\frac{1}{4}\left(\mathbf{1}+s \sigma^{3}\right) \otimes \rho^{a} \widetilde{g}_{a}^{s} .
$$

Here we made use of the chiral representation for the gamma matrices: $\quad \gamma^{0} \rightarrow \rho^{1}, \quad-i \gamma^{0} \gamma^{5} \rightarrow \rho^{2}, \quad-\gamma^{5} \rightarrow \rho^{3} \quad$ and $\quad \widetilde{S}_{z}$ $\equiv \gamma^{0} \gamma^{3} \gamma^{5} \rightarrow \sigma^{3}$, where $\rho^{a}=\left(\mathbf{1}, \rho^{i}\right)$, and $\sigma^{3}$ and $\rho^{i}$ are the
Pauli matrices. The normalization was chosen such that the component $\widetilde{g}_{0}^{s}$ corresponds to the phase space density of states of spin $s$ in the boosted frame. All of the results derived in [3] apply for the functions $\widetilde{g}_{\alpha}^{s}$ of course. From these we only need the constraint equations (here written to first order in gradients)

$$
\begin{array}{r}
k_{0} \widetilde{g}_{0}^{s}-s k_{z} \tilde{g}_{3}^{s}-m_{R} \tilde{g}_{1}^{s}-m_{I} \widetilde{g}_{2}^{s}=0 \\
k_{0} \widetilde{g}_{3}^{s}-s k_{z} \widetilde{g}_{0}^{s}+\frac{1}{2} m_{R}^{\prime} \partial_{k_{z}} \widetilde{g}_{2}^{s}-\frac{1}{2} m_{I}^{\prime} \partial_{k_{z}} \widetilde{g}_{1}^{s}=0 \\
k_{0} \widetilde{g}_{1}^{s}+\frac{s}{2} \partial_{z} \widetilde{g}_{2}^{s}-m_{R} \widetilde{g}_{0}^{s}+\frac{1}{2} m_{I}^{\prime} \partial_{k_{z}} \widetilde{g}_{3}^{s}=0 \\
k_{0} \widetilde{g}_{2}^{s}-\frac{s}{2} \partial_{z} \widetilde{g}_{1}^{s}-m_{I} \widetilde{g}_{0}^{s}-\frac{1}{2} m_{R}^{\prime} \partial_{k_{z}} \widetilde{g}_{3}^{s}=0 .
\end{array}
$$

Equations (19)-(21) allow us to express the functions $\widetilde{g}_{i}^{s}$ in terms of $\tilde{g}_{0}^{s}$ alone, while the remaining Eq. (18) contains the spectral condition for $\widetilde{g}_{0}^{s}$.

We now make an explicit connection between the solutions in the boosted and unboosted frame. To this end it is important to note that in the stationary case under study the spin in the $z$-direction remains a good quantum number also in the unboosted frame. This is mathematically expressed by the fact that the spin operator in the original frame

$$
S_{z} \equiv L^{-1}(\Lambda) \widetilde{S}_{z} L(\Lambda)=\gamma_{\|}\left[\tilde{S}_{z}-i\left(\vec{v}_{\|} \times \vec{\alpha}\right)_{z}\right]
$$

commutes with the differential operator in Eq. (10). As a consequence, even in the original frame, the problem splits into two non-mixing sectors labeled by the spin $s$. By construction the Wightman function

$$
G_{s}^{<} \equiv L(\Lambda)^{-1} \widetilde{G}_{s}^{<} L(\Lambda)
$$

in the original frame commutes with the spin operator $S_{z}$. Note that the spin $\vec{S}$ and $\vec{\alpha}=\gamma^{0} \vec{\gamma}$ transform under boosts as the magnetic and electric components of an antisymmetric tensor, respectively. This can be seen from the relations $\alpha^{i}$ $=-i \sigma^{0 i}$ and $\widetilde{S}_{i}=(1 / 2) \epsilon_{i j k} \sigma^{j k}$, where $\sigma^{\mu \nu}=(i / 2)\left[\gamma^{\mu}, \gamma^{\nu}\right]$ is the totally antisymmetric tensor.

Now, the most general decomposition for the Wightman function $G_{s}^{<}$in the unboosted frame can be written in the chiral representation as $[13,14,16]$

$$
-i \gamma^{0} G_{s}^{<}=\frac{1}{4} \sigma^{a} \otimes \rho^{b} g_{a b}^{s},
$$

where $\rho^{a}=\left(\mathbf{1}, \rho^{i}\right)$ and $\sigma^{a}=\left(\mathbf{1}, \sigma^{i}\right)[21]$. The connection between the component functions in Eqs. (24) and (17) is now found [22] by direct use of the relation (15) and the explicit form of the boost operator (13): 


$$
g_{a b}^{s}=\left(\begin{array}{cccc}
\gamma_{\|} \widetilde{g}_{0}^{s} & \widetilde{g}_{1}^{s} & \widetilde{g}_{2}^{s} & \gamma_{\|} \widetilde{g}_{3}^{s} \\
v_{1} \gamma_{\|} \widetilde{g}_{3}^{s} & s v_{2} \gamma_{\|} \widetilde{g}_{2}^{s} & -s v_{2} \gamma_{\|} \widetilde{g}_{1}^{s} & v_{1} \gamma_{\|} \widetilde{g}_{0}^{s} \\
v_{2} \gamma_{\|} \widetilde{g}_{3}^{s} & -s v_{1} \gamma_{\|} \widetilde{g}_{2}^{s} & s v_{1} \gamma_{\|} \tilde{g}_{1}^{s} & v_{2} \gamma_{\|} \widetilde{g}_{0}^{s} \\
s \widetilde{g}_{9}^{s} & s \gamma_{\|} \widetilde{g}_{1}^{s} & s \gamma_{\|} \widetilde{g}_{2}^{s} & s \widetilde{g}_{3}^{s}
\end{array}\right) .
$$

Given the transformation (25) and the constraint equations (18)-(21), we are now ready to deal with the equations of motion (10). Out of the 16 equations we are primarily interested in the 00-component, defining the kinetic properties of $g_{00}^{s}$, which corresponds to the particle number density in the phase space. Other components can always be determined from $g_{00}^{s}$ by the use of constraint equations, and their physical significance will be discussed in the next section. The relevant kinetic equation has the form

$$
\partial_{z} g_{33}^{s}-m_{R}^{\prime} \partial_{k_{z}} g_{01}^{s}-m_{I}^{\prime} \partial_{k_{z}} g_{02}^{s}=0
$$

and the constraint equation reads

$$
k_{0} g_{00}^{s}-k_{i} g_{i 3}^{s}-m_{R} g_{01}^{s}-m_{I} g_{02}^{s}=0 .
$$

In Eq. (26) we have kept terms to the second and in Eq. (27) to the first order in gradients, which suffices for the derivation of the kinetic and constraint equations accurate to order $\hbar$. Using Eq. (25) and the constraints (18)-(21) it is now a simple matter to show that we get the following kinetic equation:

$$
k_{z} \partial_{z} g_{00}^{s}-\left(\frac{1}{2}|m|^{2 \prime} \partial_{k_{z}}-\frac{s}{2 \widetilde{k}_{0}}\left(|m|^{2} \theta^{\prime}\right)^{\prime} \partial_{k_{z}}\right) g_{00}^{s}=0,
$$

and the algebraic constraint equation

$$
\left(k^{2}-|m|^{2}+\frac{s}{\widetilde{k}_{0}}\left(|m|^{2} \theta^{\prime}\right)\right) g_{00}^{s}=0,
$$

which both contain nontrivial $C P$-violating terms $\propto \theta^{\prime}$, where the angle $\theta$ corresponds to the complex phase of the mass term $m=|m|(z) e^{i \theta(z)}$.

The most important outcome of these results follows from the observation that Eq. (29) has a spectral solution [23]

$$
g_{00}^{s} \equiv \sum_{ \pm} \frac{2 \pi}{Z_{s \pm}} n_{s} \delta\left(k_{0} \mp \omega_{s \pm}\right),
$$

where $\omega_{s \pm}$ denotes the dispersion relation

$$
\omega_{s \pm}=\omega_{0} \mp s \frac{|m|^{2} \theta^{\prime}}{2 \omega_{0}\left(\omega_{0}^{2}-\vec{k}_{\|}^{2}\right)^{1 / 2}}, \quad \omega_{0}=\sqrt{\vec{k}^{2}+|m|^{2}}
$$

and $Z_{s \pm}=1 \mp s|m|^{2} \theta^{\prime} / 2\left(\omega_{0}^{2}-\vec{k}_{\|}^{2}\right)^{3 / 2}$. Because of the delta function in Eq. (30) the functions $n_{s}\left(k_{0}, k_{z}, z\right)$ are projected on-shell and become the distribution functions $f_{s+}$ and $f_{s-}$ for particles and antiparticles with spin $s$, respectively, defined by

$$
\begin{aligned}
& f_{s+} \equiv n_{s}\left(\omega_{s+}, k_{z}, z\right) \\
& f_{s^{-}} \equiv 1-n_{s}\left(-\omega_{s-},-k_{z}, z\right) .
\end{aligned}
$$

This on-shell projection proves the implicit assumption underlying the semiclassical WKB-methods, that the plasma can be described as a collection of single-particle excitations with a nontrivial space-dependent dispersion relation.

Integrating Eq. (28) over the positive and negative frequencies and taking account of Eqs. (30) and (32) we now get the following on-shell kinetic equations:

$$
v_{s \pm} \partial_{z} f_{s \pm}+F_{s \pm} \partial_{k_{z}} f_{s \pm}=0,
$$

where the quasiparticle group velocity $v_{s \pm} \equiv k_{z} / \omega_{s \pm}$ is expressed in terms of the kinetic momentum $k_{z}$ and the quasiparticle energy $\omega_{s \pm}(31)$, and the semiclassical force

$$
F_{s \pm}=-\frac{|m|^{2 \prime}}{2 \omega_{s \pm}} \pm \frac{s\left(|m|^{2} \theta^{\prime}\right)^{\prime}}{2 \omega_{0}\left(\omega_{0}^{2}-\vec{k}_{\|}^{2}\right)^{1 / 2}} .
$$

When compared with the $(1+1)$-dimensional case the sole, but significant, difference in the force is that the $C P$-violating $\theta^{\prime}$-term is enhanced by the boost-factor $\gamma_{\|}$ $=\omega_{0} /\left(\omega_{0}^{2}-\vec{k}_{\|}^{2}\right)^{1 / 2}$. When averaged over the momenta, the enhancement of the semiclassical force by the boost-factor results in a stronger $C P$-violating source in the fluid transport equations by about a factor 2 , and thus a slightly more efficient baryogenesis.

So far we have considered the Boltzmann equation (33) for a static situation (in the wall frame) with planar symmetry. It is interesting to see to what extent these restrictions can be relaxed. The essential aspect of our treatment above was the requirement of spin conservation in the $z$-direction, guaranteeing the consistency of the spin conserving decomposition $G^{<} \rightarrow G_{s}^{<}$of the Wigner function. This requirement can be formally expressed by the condition

$$
\left[S_{z}, \mathcal{D}\right] G_{s}^{<}=0,
$$

where $\mathcal{D}$ is the derivative operator in Eq. (7), and the commutator evaluates to

$$
\left[\vec{S}_{z}, \mathcal{D}\right]=-\gamma_{\|} \gamma^{0} \vec{v}_{\|} \times \partial_{\vec{x}_{\|}}+\vec{\gamma}_{\|} \times \gamma_{\|}\left(\partial_{\vec{x}_{\|}}+\vec{v}_{\|} \partial_{t}\right)
$$

where $\vec{\gamma}_{\|}=\left(\gamma^{1}, \gamma^{2}, 0\right)$. The condition (35) is clearly satisfied when the parameter dependences of the Wigner function are of the form [24]

$$
G_{s}^{<}=G_{s}^{<}\left(k_{\mu} ; z, t-\vec{v}_{\|} \cdot \vec{x}_{\|}\right) .
$$

For static, planar symmetric solutions studied so far the commutation condition is satisfied trivially, because for them $\partial_{t} G^{<}=\partial_{x_{\|}} G^{<}=0$. We note that in the $1+1$ frame (in which $\vec{k}_{\|}=0$ ) our result (37) is particularly simple and intuitive. In this frame $\partial_{\tilde{t}}$ is spin conserving, while $\widetilde{\partial}_{\tilde{x}_{\|}}$violates spin, and thus the only off-diagonal contributions in the equation $\widetilde{\mathcal{D}} \widetilde{G}_{s}^{<}=0$ are the $\widetilde{\partial}_{\vec{x}_{\|}}$-derivatives, leading to the requirement 
$\widetilde{\partial}_{\vec{x} \|} \widetilde{g}_{a}^{s}=0$. This is completely consistent with Eq. (37), which in the $1+1$ frame reduces to $\widetilde{G}_{s}^{<}=\widetilde{G}_{s}^{<}\left(\widetilde{k}_{\mu} ; z, \widetilde{t}\right)$.

Following the procedure outlined earlier in this section one can show that there are no new corrections to the constraint equation (29), implying that the transients (37) respect the simple algebraic on-shell condition. The kinetic equation (33) now generalizes to

$$
\partial_{t} f_{s \pm}+\vec{v}_{s \pm} \cdot \partial_{x} f_{s \pm}+F_{s \pm} \partial_{k_{z}} f_{s \pm}=0
$$

where $\vec{v}_{s \pm} \equiv \vec{k} / \omega_{s \pm}$, and the distribution function satisfies $f_{s \pm}=f_{s \pm}\left(k_{\mu} ; z, t-\vec{v}_{\|} \cdot \vec{x}_{\|}\right)$, where now $\vec{v}_{\|} \equiv \vec{k}_{\|} / \omega_{s \pm}$. We shall assume this to be satisfied when discussing the current continuity equations in the next section.

\section{FERMIONIC CURRENTS AND CP-VIOLATING SOURCES}

In order to shed light on the physical significance of the various components of the matrix $G_{s}^{<}$, it is instructive to consider the fermionic currents

$$
\begin{aligned}
\langle\bar{\psi}(x) \Gamma \psi(x)\rangle & =-\int \frac{d^{4} k}{(2 \pi)^{4}} \operatorname{Tr}\left[\Gamma i G^{<}(x, k)\right] \\
\Gamma & =\left(\mathbf{1}, \boldsymbol{\gamma}^{5}, \gamma^{\mu}, \gamma^{5} \gamma^{\mu}, \sigma^{\mu \nu}=\frac{i}{2}\left[\gamma^{\mu}, \gamma^{\nu}\right]\right) .
\end{aligned}
$$

By direct computation one sees that $g_{01}^{s}$ and $g_{02}^{s}$ correspond to a scalar and pseudoscalar density, respectively, while $\left(g_{00}^{s}, g_{i 3}^{s}\right)$ forms a 4-vector, and $\left(g_{03}^{s}, g_{i 0}^{s}\right)$ a 4-pseudovector. Finally, $g_{i 1}^{s}$ and $g_{i 2}^{s}$ are components of an antisymmetric tensor, and so they transform like the magnetic and electric field, respectively. These transformation properties could actually have been used as an alternative and elegant way to infer the relation between the boosted and nonboosted frames in the previous section.

Let us now look at the vector and pseudovector currents in a little more detail. By writing $g_{a b}^{s}$ in terms of $g_{00}^{s}$ and dropping the total derivative terms we find

$$
\begin{aligned}
& j_{s}^{\mu}=\int \frac{d^{4} k}{(2 \pi)^{4}}(1 ; \vec{v}) g_{00}^{s} \\
& j_{5 s}^{\mu}=\int \frac{d^{4} k}{(2 \pi)^{4}}\left(1 ; \vec{v}_{\|}, \frac{1}{\gamma_{\|}^{2} v_{s}}\right) s v_{s} \gamma_{\|} g_{00}^{s} .
\end{aligned}
$$

These expressions are clearly the expected generalizations with appropriate boost factors of the $(1+1)$-dimensional currents derived in [3].

It is straightforward to show from the equations of motion, that the vector current divergence is conserved, and the axial vector current has the usual form (since we do not treat the gauge fields here, the axial vector current is taken to be nonanomalous; for an account of the anomaly see [8])

$$
\begin{aligned}
\partial_{\mu} j_{s}^{\mu} & =0 \\
\partial_{\mu} j_{5 s}^{\mu} & =2 i m_{R}\left\langle\bar{\psi}_{s} \gamma^{5} \psi_{s}\right\rangle-2 m_{I}\left\langle\bar{\psi}_{s} \psi_{s}\right\rangle .
\end{aligned}
$$

If we define the fluid density [25] $n_{s \pm}$ and fluid velocity moments as [26]

$$
\begin{gathered}
n_{s+} \equiv \int_{+} \frac{d^{4} k}{(2 \pi)^{4}} g_{00}^{s} \\
\left\langle v_{s+}^{p}\right\rangle \equiv \frac{1}{n_{s+}} \int_{+} \frac{d^{4} k}{(2 \pi)^{4}}\left(\frac{k_{z}}{k_{0}}\right)^{p} g_{00}^{s},
\end{gathered}
$$

then Eqs. (42)-(43) can be shown to correspond to the two lowest velocity moments of the Boltzmann equation (38):

$$
\begin{gathered}
\partial_{t} n_{s \pm}+\partial_{z}\left(n_{s \pm}\left\langle v_{s \pm}\right\rangle\right)=0, \\
\partial_{t}\left(n_{s \pm}\left\langle v_{s \pm}\right\rangle\right)+\partial_{z}\left(n_{s \pm}\left\langle v_{s \pm}^{2}\right\rangle\right)=S_{s \pm} .
\end{gathered}
$$

The quantity $S_{s \pm}$ is related to the baryogenesis source and is given by the average over the semiclassical force (34) divided by $k_{0}$ :

$$
S_{s \pm}=-\frac{1}{2}|m|^{2} \mathcal{I}_{2 s \pm} \pm \frac{1}{2} s\left(|m|^{2} \theta^{\prime}\right)^{\prime} \mathcal{I}_{3 s \pm},
$$

and

$$
\mathcal{I}_{p s+} \equiv \int_{+} \frac{d^{4} k}{(2 \pi)^{4}} \frac{g_{00}^{s}}{k_{0}^{p}}
$$

It should be stressed that while Eqs. (45), (46) are illustrative in relating the baryogenesis source in moment equations to the non-conservation of the axial vector current, they are somewhat formal, and not directly suitable for actual calculations. For practical baryogenesis calculations it is convenient to treat the Boltzmann equation (38) in linear response approximation with respect to deviations from thermal equilibrium

$$
\delta f_{s \pm} \equiv f_{s \pm}-f_{0 s \pm}, \quad f_{0 s \pm}=\frac{1}{e^{\beta \gamma_{w}\left(\omega_{s \pm}+v_{w} k_{z}\right)}+1}
$$

where $f_{0 s^{ \pm}}$is the equilibrium distribution function in the wall frame, $\beta=1 / T, v_{w}$ is the wall velocity and $\gamma_{w}$ is the corresponding boost factor. Working to linear order in $v_{w}$ we can rewrite Eq. (38) as

$\left(\partial_{t}+\vec{v}_{s \pm} \cdot \partial_{x}+F_{s \pm} \partial_{k_{z}}\right) \delta f_{s \pm}+v_{w} F_{s \pm}\left(\partial_{\omega} f_{\omega}\right)_{\omega=\omega_{s \pm}}=0$, 
with $f_{\omega} \equiv 1 /\left(e^{\beta \omega}+1\right)$. Equation (50) implies the following kinetic equation for the $C P$-violating density $\delta f_{s} \equiv \delta f_{s+}$ $-\delta f_{s-}$ :

$$
\begin{aligned}
\left(\partial_{t}+\right. & \left.\frac{\vec{k}}{\omega_{0}} \cdot \partial_{x}-\frac{|m|^{2 \prime}}{2 \omega_{0}} \partial_{k_{z}}\right) \delta f_{s} \\
= & -v_{w}\left(\delta F_{s}\left(\partial_{\omega} f_{\omega}\right)_{\omega_{0}}+F_{0} \delta \omega_{s}\right. \\
& \left.\times\left[\left(\frac{\partial_{\omega} f_{\omega}}{\omega}\right)_{\omega_{0}}-\left(\partial_{\omega}^{2} f_{\omega}\right)_{\omega_{0}}\right]\right),
\end{aligned}
$$

where we kept only the leading second order terms in gradients, and

$$
\begin{gathered}
F_{0} \equiv-\frac{|m|^{2 \prime}}{2 \omega_{0}} \\
\delta \omega_{s} \equiv s \frac{|m|^{2} \theta^{\prime}}{\omega_{0} \tilde{\omega}_{0}} \\
\delta F_{s} \equiv F_{s+}-F_{s-}=s \frac{\left(|m|^{2} \theta^{\prime}\right)^{\prime}}{\omega_{0} \tilde{\omega}_{0}},
\end{gathered}
$$

where $\tilde{\omega}_{0}=\left(\omega_{0}^{2}-\vec{k}_{\|}^{2}\right)^{1 / 2}$. Equation (51) displays two very important features not evident in Eqs. (45), (46): the $C P$-violating perturbation $\delta f_{s}$ is only sourced by terms second order in derivatives (first order in $\hbar$ ) and the source is suppressed by the wall velocity.

To get equations suitable for baryogenesis calculations it would be necessary to include collision terms in Eq. (51), which are responsible for all thermalization and transport processes. While treating collisions is beyond the scope of this paper, let us nevertheless mention that an approximate solution for Eq. (51) with collisions is typically found by taking moments of the kinetic equation, leading to a set of fluid equations for the moments. In order to get a good approximation while using only a few lowest moments, it is important to parametrize $\delta f_{s}$ such that the parametrization models the actual solution in the best possible way. Particularly suitable and often used is the fluid ansatz parametrized by the chemical potential and a perturbation that is an odd function of the momentum. The chemical potential then captures an excess of particles formed at the phase boundary front, while the latter parametrizes the anisotropic bulk motion of the fluid. For a more detailed account of the fluid equations we refer the reader to Refs. [9,11,12,27].

We finally note that the continuity equations for the vector and axial vector current (45), (46) are equivalent to the two lowest order moments of the Boltzmann equation (51) [3], and hence also to the fluid equations.

\section{WKB METHOD}

For the sake of comparison we shortly review the derivation of the semiclassical transport equations via the WKB- method [9-11], in which the dispersion relations of the single particle excitations are derived directly from the Dirac equation (4) in the gradient expansion. In the wall frame the mass (2) is only a function of $z, m(z)=|m(z)| e^{i \theta(z)}$, and therefore the energy and momentum parallel to the wall are conserved quantities. We can then seek the solutions of the form $\psi(x)=\exp \left(-i k_{0} t+i \vec{k}_{\|} \cdot \vec{x}_{\|}\right) \psi_{k_{0} \vec{k}_{\|}}(z)$, which gives

$$
\left(\gamma^{0} k_{0}-\vec{\gamma} \cdot \vec{k}_{\|}-i \gamma^{3} \partial_{z}-m_{R}-i \gamma^{5} m_{I}\right) \psi_{k_{0} \vec{k}_{\|}}(z)=0
$$

As in the previous section, we can use the Lorentz boost $\Lambda$ (13) to get rid of the $\vec{\gamma} \cdot \vec{k}_{\|}$term in Eq. (53). The remaining part of the Dirac operator then commutes with the spin operator $\widetilde{S}_{z}(16)$, indicating that the spin $s$ in the $z$-direction is a good quantum number, and one can write the boosted wave function $\widetilde{\psi}_{\widetilde{k}_{0}}=L(\Lambda) \psi_{k_{0} \vec{k}_{\|}}$as a direct product of $2 \times 2$ spinors,

$$
\widetilde{\psi}_{\tilde{k}_{0}} \equiv \widetilde{\psi}_{\tilde{k}_{0}}^{s} \otimes \chi_{s}, \quad \widetilde{\psi}_{\tilde{k}_{0}}^{s}=\left(\begin{array}{c}
L_{s} \\
R_{s}
\end{array}\right)
$$

where $\chi_{s}$ is the eigenspinor of the spin operator (16) in the $2 \times 2$ spinor space, $\sigma^{3} \chi_{s}=s \chi_{s}$, and $\widetilde{k}_{0}=\operatorname{sgn}\left[k_{0}\right]\left(k_{0}^{2}-k_{\|}^{2}\right)^{1 / 2}$ as usual. With this decomposition Eq. (53) simplifies to the following two equations for the left and right chiral states in the boosted frame:

$$
\begin{aligned}
& \left(\widetilde{k}_{0}-i s \partial_{z}\right) L_{s}=m R_{s} \\
& \left(\widetilde{k}_{0}+i s \partial_{z}\right) R_{s}=m^{*} L_{s} .
\end{aligned}
$$

Our analysis so far differs from the ones in the literature in that we have discussed more explicitly the boost and its relation to the spin-decomposition. The following steps in the analysis are quite standard, and we refer the reader for example to $[8,9]$ for more details. Solving $R_{s}$ in terms of $L_{s}$ in Eq. (55) and then introducing the WKB-parametrization

$$
L_{s}=u_{s} e^{i \int^{z} k_{s} d z^{\prime}}
$$

one can write down a set of two coupled second order equations for $u_{s}$ and $k_{s}$. Solving these equations to second order in gradients gives the dispersion relation

$$
k_{s}=p_{0}+s_{\mathscr{P}} \frac{\left(s k_{0}+p_{0}\right) \theta^{\prime}}{2 p_{0}}
$$

where $p_{0}=\operatorname{sgn}\left(k_{s}\right) \sqrt{\widetilde{k}_{0}^{2}-|m|^{2}}$ and $s_{\mathscr{C P}}=1(-1)$ for particles (antiparticles). Remembering that [28] $\widetilde{k}_{0}^{2}=k_{0}^{2}-k_{\|}^{2}$ and inverting Eq. (57) one can write the energy in the unboosted frame as

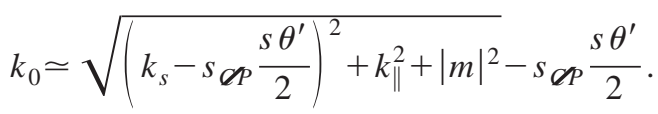


From this expression for the energy we can finally compute the physical momentum, which is defined in terms of the group velocity $v_{g}=k_{0}\left(\partial_{k_{s}} k_{0}\right)_{z}$ :

$$
k_{z} \equiv k_{0} v_{g}=p_{0}+s_{\mathscr{C P}} \frac{s|m|^{2} \theta^{\prime}}{2 p_{0} \widetilde{k}_{0}}
$$

and the corresponding physical force $F_{z}=\dot{k}_{z}=\omega_{s \pm} d v_{s \pm} / d t$ :

$$
F_{z}=-\frac{|m|^{2 \prime}}{2 k_{0}}+s_{\mathscr{C P}} \frac{s\left(|m|^{2} \theta^{\prime}\right)^{\prime}}{2 k_{0} \widetilde{k}_{0}} .
$$

These results are in complete agreement with those found in Sec. II, completing the explicit proof of correctness of the semiclassical limit in the present paper.

Let us finally point out that the kinetic equations could be formulated in terms of the canonical momentum (57), as it was done originally in $[11,29]$. The transformation that relates the kinetic and canonical momenta is noncanonical:

$$
k_{z}=k_{s}\left(1 \mp \frac{s \theta^{\prime}}{2\left(k_{s}^{2}+|m|^{2}\right)^{1 / 2}}\right) .
$$

The advantage of the canonical formulation is that it allows for a Hamiltonian formulation of kinetic equations, while the disadvantage is that the canonical momentum and energy do not transform as a 4-vector under Lorentz boosts. This makes the formulation of fluid equations cumbersome, and so far it has not been done with due care in the literature [10-12,29].

\section{APPLICATIONS TO SUPERSYMMETRIC MODELS}

When studying electroweak baryogenesis in supersymmetric models an important source comes from $C P$-violating dynamics of charginos in the presence of a bubble wall. In this case a formalism that describes the dynamics of mixing fermions is required. Introducing the mixing does not bring any extra complications with respect to extension to $3+1$ dimensions and hence, proceeding just as in KPSW-I [3], we find the semiclassical Boltzmann equation

$$
\partial_{t} f_{s i \pm}+\vec{v}_{s i \pm} \cdot \partial_{x}^{-} f_{s i \pm}+F_{s i \pm} \partial_{k_{z}} f_{s i \pm}=0
$$

where $f_{s i+}$ and $f_{s i-}$ are the distribution functions for fermions and antifermions of spin $s$, respectively, written in the propagating basis in which the mass matrix is diagonal. As it was the case in Eq. (38), $f_{s i \pm}$ 's are assumed to satisfy the condition $f_{s i \pm}=f_{s i \pm}\left(k_{\mu} ; z, t-\vec{v}_{\|} \cdot \vec{x}_{\|}\right)$.

The group velocity of the $i$ th quasiparticle eigenstate equals $\vec{v}_{s i \pm}=\vec{k} / \omega_{s i \pm}$ and the $C P$-violating semiclassical force reads

$$
F_{s i \pm}=-\frac{\left|M_{i}\right|^{2 \prime}}{2 \omega_{s i \pm}} \pm \frac{s\left(\left|M_{i}\right|^{2} \Theta_{i}^{\prime}\right)^{\prime}}{2 \omega_{0 i}\left(\omega_{0 i}^{2}-\vec{k}_{\|}^{2}\right)^{1 / 2}}
$$

where $\left|M_{i}\right|^{2} \Theta_{i}^{\prime}$ is a diagonal matrix which can be computed from the original mass matrix and the rotation matrix $U$ diagonalizing $M M^{\dagger}$ as follows:

$$
\left|M_{d}\right|^{2} \Theta^{\prime}=\operatorname{Im}\left(U M M^{\prime \dagger} U^{\dagger}\right)_{d} .
$$

For example, in the case of charginos the mass term reads

$$
\bar{\Psi}_{R} M \Psi_{L}+\text { H.c., }
$$

where $\Psi_{R}=\left(\widetilde{W}_{R}^{+}, \widetilde{h}_{1, R}^{+}\right)^{T}$ and $\Psi_{L}=\left(\widetilde{W}_{L}^{+}, \widetilde{h}_{2, L}^{+}\right)^{T}$ are chiral fields consisting of $w$-inos and Higgsinos. The mass matrix is conveniently parametrized as

$$
M=\left(\begin{array}{cc}
m_{2} & g H_{2}^{*} \\
g H_{1}^{*} & \mu
\end{array}\right),
$$

where $H_{1}=h_{1} e^{i \theta_{1}}$ and $H_{2}=h_{2} e^{i \theta_{2}}$ are the Higgs field vacuum expectation values (VEV's) and $\mu$ and $m_{2}$ are the soft supersymmetry breaking parameters. For a realistic choice of parameters there is no spontaneous $C P$-violation in the minimal supersymmetric standard model (MSSM), so to a good approximation we can take the Higgs VEV's to be real $[30,31]$. In this case $[3,9,29]$

$$
\left|M_{i}\right|^{2} \Theta_{i}^{\prime} \Rightarrow m_{ \pm}^{2} \Theta_{ \pm}^{\prime}=\mp \frac{g^{2}}{\Lambda} \operatorname{Im}\left(\mu m_{2}\right)\left(h_{1} h_{2}\right)^{\prime},
$$

where $\Theta_{+}\left(\Theta_{-}\right)$corresponds to the Higgsino-like state when $|\mu|>\left|m_{2}\right|\left(|\mu|<\left|m_{2}\right|\right)$, and $\Lambda=m_{+}^{2}-m_{-}^{2}$ is the mass splitting of the chargino mass eigenstates $[3,9,29]$. $C P$-violation is here mediated via the parameters $\mu, m_{2}$ and may be large [32]. Yet another interesting $C P$-violating source arises when the Higgs condensate develops a $C P$-violating phase, as it is in the case of the NMSSM [12] (for details of computation see [3]).

\section{CONCLUSIONS AND DISCUSSION}

We have presented a first principles derivation of the semiclassical limit of transport equations for a collisionless gas of fermions in a spatially varying background. Our analysis is based on the gradient expansion in the relative coordinate of the Kadanoff-Baym equations of motion [5] for the out-of-equilibrium two point function $G^{<}$. The most crucial step in our derivation was to show that to first order in $\hbar$ the Wigner transformed function $G^{<}$has a spectral solution. The transport equations for the corresponding on-shell excitations are shown to reduce to the usual Liouville form, and contain a $C P$-violating semiclassical force of order $\hbar$. We also showed how the same force can be obtained by WKB methods $[9-12,29]$. The force is suitable for baryogenesis calculations based on the charge transport picture [33] at a first order electroweak phase transition.

The present work extends our earlier $(1+1)$-dimensional results [3] to the more realistic case of a planar phase transition wall in $3+1$ dimensions, relevant for EWBG. The problem becomes analytically tractable only upon realizing that the spin orthogonal to the moving planar wall remains a 
good quantum number for particles moving in arbitrary directions with respect to the wall. Thanks to this conserved quantity the problem can be reduced to the dynamics of one spin dependent distribution function for fermions, and one for antifermions. We assumed planar symmetry and stationarity appropriate for EWBG. For completeness we also discussed how our formalism can be extended to include particular time and space dependent transients which conserve spin.

The spectral solution for $G^{<}$can only be found up to order $\hbar$. Beyond order $\hbar$ the spectral decomposition ansatz is not consistent and the transport equations do not have the semiclassical Liouville limit. It is therefore very fortunate that in at least some of the most interesting cases [6] the phase transition walls are rather wide $l_{\text {wall }} \gg l_{\mathrm{dB}}$, where $l_{\mathrm{dB}}$ is the de Broglie wavelength $\sim 1 / T$ of a particle in the plasma, and so the semiclassical equation is expected to be a reasonable approximation.

The case of mixing fermions is relevant for example in supersymmetric baryogenesis scenarios, in which baryogen- esis can be mediated by mixing of charginos or neutralinos and other species. Supersymmetric models, in contrast to the minimal version of the standard model [34], are shown to be viable candidates for EWBG [35]. We therefore derived explicitly the $C P$-violating force for a general case of mixing fermions and for charginos in particular. With this, the present work covers the proof of the semiclassical methods used for baryogenesis calculations in the literature so far $[9,12]$. The directions for further work include the inclusion of the self-consistent gauge (hyperelectric) fields [8] in 3 +1 dimensions and the study of interactions, which introduce new collisional baryogenesis sources [7].

\section{ACKNOWLEDGMENTS}

We wish to thank Michael Joyce for earlier collaboration on the Schwinger-Keldysh formalism for fermions, and Dietrich Bödeker for raising a doubt about the validity of the spectral decomposition ansatz as a general tool for the Kadanoff-Baym equations.
[1] V.A. Kuzmin, V.A. Rubakov, and M.E. Shaposhnikov, Phys. Lett. 155B, 36 (1985)

[2] A.G. Cohen, D.B. Kaplan, and A.E. Nelson, Phys. Lett. B 245, 561 (1990); A.G. Cohen, D.B. Kaplan, and A.E. Nelson, Nucl. Phys. B349, 727 (1991).

[3] K. Kainulainen, T. Prokopec, M.G. Schmidt, and S. Weinstock, J. High Energy Phys. 06, 031 (2001).

[4] J. Schwinger, J. Math. Phys. 2, 407 (1961); L.V. Keldysh, Zh. Éksp. Teor. Fiz. 47, 1515 (1964) [Sov. Phys. JETP 20, 1018 (1964)].

[5] L.P. Kadanoff and G. Baym, Quantum Statistical Mechanics (Benjamin Press, New York, 1962).

[6] J.M. Moreno, M. Quiros, and M. Seco, Nucl. Phys. B526, 489 (1998); J.M. Cline, G.D. Moore, and G. Servant, Phys. Rev. D 60, 105035 (1999); G.D. Moore, J. High Energy Phys. 03, 006 (2000); J.M. Cline and G.D. Moore, Phys. Rev. Lett. 81, 3315 (1998).

[7] K. Kainulainen, T. Prokopec, M.G. Schmidt, and S. Weinstock, in Proceedings of COSMO-01, hep-ph/0201245.

[8] K. Kainulainen, T. Prokopec, M.G. Schmidt, and S. Weinstock, in Proceedings of COSMO-01, hep-ph/0201293.

[9] J.M. Cline and K. Kainulainen, Phys. Rev. Lett. 85, 5519 (2000); J.M. Cline, M. Joyce, and K. Kainulainen, J. High Energy Phys. 07, 018 (2000); K. Kainulainen, in Proceedings of COSMO 99, Trieste, Italy, 1999 (World Scientific, Singapore, 2000), p. 383.

[10] M. Joyce, T. Prokopec, and N. Turok, Phys. Rev. Lett. 75, 1695 (1995); 75, 3375(E) (1995).

[11] M. Joyce, T. Prokopec, and N. Turok, Phys. Rev. D 53, 2958 (1996).

[12] S.J. Huber and M.G. Schmidt, Nucl. Phys. B606, 183 (2001); "Baryogenesis at the electroweak phase transition for a SUSY model with a gauge singlet," hep-ph/0011059.

[13] H.T. Elze, M. Gyulassy, and D. Vasak, Nucl. Phys. B276, 706 (1986); D. Vasak, M. Gyulassy, and H.T. Elze, Ann. Phys. (N.Y.) 173, 462 (1987).
[14] I. Bialynicki-Birula, P. Gornicki, and J. Rafelski, Phys. Rev. D 44, 1825 (1991).

[15] P. Zhuang and U. Heinz, Phys. Rev. D 57, 6525 (1998).

[16] G.R. Shin and J. Rafelski, Ann. Phys. (N.Y.) 243, 65 (1995).

[17] P. Zhuang and U. Heinz, Phys. Rev. D 53, 2096 (1996).

[18] K. Kainulainen, T. Prokopec, M. G. Schmidt, and S. Weinstock (in preparation).

[19] To what extent the assumption of stationarity can be relaxed is discussed at the end of this section.

[20] To get a flavor of how the complete set of equations looks, we refer to Refs. [16,17].

[21] It is easy to show that $\rho^{a} \otimes \sigma^{b}$ span the full $(3+1)$-dimensional Clifford algebra.

[22] An alternative way to derive this connection would be to identify the transformation properties of $g_{a b}^{s}$ by considering the fermionic currents, see Sec. III.

[23] The normalization of $\widetilde{G}^{<}$in Eq. (17), combined with the spectral sum rule $i \gamma^{0} \int d k_{0}\left(G^{>}-G^{<}\right)=2 \pi \mathbf{1}$, fixes the normalization in Eq. (30) unambiguously. It differs by a factor of 4 from our definition in [3], which was thus not entirely consistent.

[24] We have checked that, when Eq. (37) is inserted in $\mathcal{D} G_{s}^{<}=0$, one obtains a fully consistent set of 16 equations for the components $\tilde{g}_{a}^{s}(a=0 \ldots 3)$.

[25] $n_{s \pm}$ should not be confused with the phase space density $n_{s}$ in Eq. (30).

[26] Here we defined integration over the positive frequencies. To obtain equations for antiparticles one integrates over negative frequencies taking into account the appropriate thermal equilibrium limit in Eq. (32).

[27] G.D. Moore and T. Prokopec, Phys. Rev. D 52, 7182 (1995).

[28] The transform back from $\widetilde{k}_{0}$ to $k_{0}$ was not effected in the derivation presented in [9], leading to a missing $\gamma_{\|}$-factor in the $C P$-violating force. The gamma-factor was included in [36] however.

[29] J.M. Cline, M. Joyce, and K. Kainulainen, Phys. Lett. B 417, 
79 (1998); 448, 321(E) (1998).

[30] S.J. Huber, P. John, M. Laine, and M.G. Schmidt, Phys. Lett. B 475, 104 (2000).

[31] S.J. Huber, P. John, and M.G. Schmidt, Eur. Phys. J. C 20, 695 (2001)

[32] A. Pilaftsis and C.E. Wagner, Nucl. Phys. B553, 3 (1999).

[33] A.G. Cohen, D.B. Kaplan, and A.E. Nelson, Phys. Lett. B 245, 561 (1990); A.E. Nelson, D.B. Kaplan, and A.G. Cohen, Nucl. Phys. B373, 453 (1992).

[34] K. Kajantie, M. Laine, K. Rummukainen, and M. Shaposhnikov, Nucl. Phys. B493, 413 (1997); Phys. Rev. Lett. 77, 2887
(1996); Nucl. Phys. B466, 189 (1996).

[35] M. Carena, M. Quiros, and C.E. Wagner, Phys. Lett. B 380, 81 (1996); Nucl. Phys. B524, 3 (1998); J.M. Cline and K. Kainulainen, ibid. B482, 73 (1996); B510, 88 (1998); M. Laine, ibid. B481, 43 (1996); B548, 637(E) (1996); M. Losada, Phys. Rev. D 56, 2893 (1997); D. Bödeker, P. John, M. Laine, and M.G. Schmidt, Nucl. Phys. B497, 387 (1997); M. Laine and K. Rummukainen, ibid. B535, 423 (1998); B597, 23 (2001).

[36] J.M. Cline, M. Joyce, and K. Kainulainen, "Erratum for Supersymmetric electroweak baryogenesis," hep-ph/0110031. 